\title{
Predictors of in-hospital mortality in coronary artery dissection: Findings from the National Inpatient Sample 2009-2010
}

\author{
Jalaj Garg ${ }^{1}$, Parasuram Krishnamoorthy ${ }^{2}$, Chandrasekar Palaniswamy ${ }^{1}$, \\ Ambarish Pandey ${ }^{3}$, Hasan Ahmad ${ }^{1}$ \\ ${ }^{1}$ Department of Medicine, Division of Cardiology, Westchester Medical Center, \\ New York Medical College, NY, United States \\ ${ }^{2}$ Department of Medicine, Englewood Hospital and Medical Center, NJ, United States \\ ${ }^{3}$ Department of Medicine, Division of Cardiology, University of Texas, Texas, United States
}

\begin{abstract}
Background: The pathophysiology of coronary artery dissection (CD) remains poorly understood and little is known about the factors predicting mortality in these patients. We aimed to study the epidemiology of CD and predictors of mortality in these patients.

Methods: All patients diagnosed with CD in the Nationwide Inpatient Sample 2009-2010 database using International Classification of Diseases ninth revision 414.12 were included in the study. Chronic conditions included in the analysis were diabetes mellitus, hypertension, hyperlipidemia, coronary artery disease (CAD), obesity, alcohol use, smoking, heart failure and ventricular arrhythmias. Non-cardiovascular conditions were connective tissue disorders, fibromuscular dysplasia, Ehlers-Danlos syndrome, Marfan's syndrome, sarcoidosis, Crohn's disease, polycystic kidney disease, rheumatoid arthritis, vasculitis including giant cell arteritis, polyarteritis nodosa and Takayasu's disease, cocaine use, early or premature labor.
\end{abstract}

Results: The prevalence of CD in the United States was 0.02\% ( $n=11,255)$, based on the hospital admissions reviewed in the database. The mean age was 63.25 years with women (64.62 years) being older than men (62.25 years) ( $p<0.001)$. In-hospital mortality rate was $4.2 \%$, with women (5.5\%) having higher mortality than men (3.2\%) $(p=0.009)$. Ventricular arrhythmias (OR 5.86, $p<0.001)$ predicted higher mortality, while hyperlipidemia (OR 0.26, $p<0.001)$ and $C A D(O R$ 0.31, $p=0.001)$ predicted lower mortality in multivariate analysis.

Conclusions: Our study demonstrated that CD was more prevalent in men but women had higher mortality than men. Age, heart failure and ventricular arrhythmias were independent predictors of increased mortality but hyperlipidemia CAD predicted lower mortality in patients with CD. (Cardiol J 2015; 22, 2: 135-140)

Key words: coronary artery dissection, risk factors, predictors of mortality, arrhythmias

Address for correspondence: Jalaj Garg, MD, Department of Medicine, Division of Cardiology, Westchester Medical Center, New York Medical College, Valhalla, NY 10595, USA, tel: 585-766-0898, fax: 914-493-1414, e-mail: garg.jalaj@yahoo.com AND

Parasuram Krishnamoorthy, MD, Department of Medicine, Englewood Hospital and Medical Center, Englewood, NJ 07631, USA, tel: 732501 5100, fax: 201-894-0839, e-mail: parasumk@yahoo.com

This paper is not under consideration elsewhere. None of the paper's contents have been previously published except in an American Heart Association 2013 abstract.

Received: 28.03.2014 Accepted: 10.05.2014 


\section{Introduction}

Coronary artery dissection (CD) is rare with a prevalence of $0.07 \%$ to $1.1 \%$. The first case of $\mathrm{CD}$ was reported in 1931 [1] and about 440 cases of spontaneous coronary artery dissection (SCD) have been reported in the literature since then [2]. It is characterized by separation of the coronary arterial wall due to bleeding within the wall either from an intimal tear or a medial hematoma $[3,4]$. Both processes result in compromised flow either from multiple lumens or flaps in the case of an intimal tear or luminal narrowing from the intramural hematoma, resulting in compromised antegrade blood flow. The clinical presentation is that of an acute coronary syndrome (ACS) which includes angina, non-ST elevation myocardial infarction (NSTEMI) or ST-segment elevation myocardial infarction (STEMI) and ventricular fibrillation and/or sudden cardiac death [5-7]. CD can be SCD, traumatic (as a complication of coronary angiography) [8, 9], and either atherosclerotic or non-atherosclerotic. Non-atherosclerotic SCD is associated with fibromuscular dysplasia (FMD), inflammatory and connective tissue disorders, emotional stress, exertion, post partum state and is more common in younger women $[3,4,10]$. Typically CD involves a single coronary artery, however multi vessel coronary artery dissection has also been reported [3].

$\mathrm{CD}$ and especially SCD remain under diagnosed and the pathophysiology is still poorly understood. The reasons include under recognition of CD as a cause of STEMI and arrhythmia related sudden cardiac death, misdiagnosis of chest pain in younger females with SCD as non-cardiac chest pain and incorrectly characterizing an intramural hematoma as spasm or atherosclerotic plaque during angiography. In addition little is known about the factors predicting mortality in $\mathrm{CD}$, which till now were largely limited to case reports and small case series. The objective of our analysis was to determine the epidemiology and predictors of mortality in patients with CD.

\section{Methods}

We accessed the Nationwide Inpatient Sample (NIS) database for the period 2009 to 2010 from the Healthcare Cost and Utilization Project of the Agency for Healthcare Research and Quality, in Rockville, MD. It is the largest all-payer hospitaliza- tion database representing up to 8 million hospital stays from 1,000 hospitals in the United States. The data represents $20 \%$ of all inpatient admissions to non-federal hospitals in the United States. All patients with primary and all listed diagnoses of $\mathrm{CD}$ in the NIS 2009-2010 database using International Classification of Diseases 9 (ICD-9) 414.12 were included in the study. As the ICD-9 code was restricted only to the diagnosis of $\mathrm{CD}$ and no other codes exist, the type of $\mathrm{CD}$ (spontaneous, atherosclerotic, nonatherosclerotic or iatrogenic) was uncertain. All hospitalizations of any patient aged 18 years or older were included in the analysis. Age was represented as a continuous variable while race was categorized as white, black, Asian, Hispanic, native American or others. We weighted hospital and discharge data to generate national estimates.

\section{Outcomes}

The primary outcomes of our study were predictors and prevalence of in-hospital mortality. We assessed the demographics and the prevalence of chronic conditions in patients with $\mathrm{CD}$. Chronic conditions included diabetes mellitus, hypertension, hyperlipidemia, obesity, coronary artery disease (CAD), smoking and alcohol use. Non-cardiovascular conditions analyzed were connective tissue disorders, fibromuscular dysplasia, Ehlers-Danlos syndrome, Marfan's syndrome, sarcoidosis, Crohn's disease, cocaine use, polycystic kidney disease, rheumatoid arthritis, vasculitis including giant cell arteritis, polyarteritis nodosa and Takayasu's disease and early or premature labor. We also determined the prevalence of heart failure (HF) and ventricular arrhythmia (refer to supplement for ICD-9 codes used).

\section{Statistical analysis}

We analyzed the database from 2009 to 2010. Categorical variables were represented as percentages (\%), while continuous variables were represented as mean \pm standard deviation. Univariate regression analysis was done to determine predictors of mortality in CD. Including traditional risk factors and other factors that predicted mortality in univariate analysis, stepwise multimodel logistic regression was performed. A p value less than 0.05 was considered statistically significant. All statistical analysis was performed using STATA 10.0, Statacorp, College Station, TX. The study did not have any outside sponsorship or funding. 
Table 1. Baseline demographic and clinical characteristics stratified by gender.

\begin{tabular}{|c|c|c|c|c|}
\hline Variable* & $\begin{array}{c}\text { Total } \\
(\mathrm{n}=11,255)\end{array}$ & $\begin{array}{c}\text { Males } \\
(n=6,498)\end{array}$ & $\begin{array}{c}\text { Females } \\
(\mathrm{n}=4,757)\end{array}$ & $\mathbf{P} * *$ \\
\hline Age & $63.3 \pm 0.3$ & $62.3 \pm 0.3$ & $64.6 \pm 0.5$ & $<0.001$ \\
\hline Length of stay & $4.5 \pm 0.1$ & $4.1 \pm 0.1$ & $4.9 \pm 0.2$ & 0.001 \\
\hline Mortality & 4.2 & 3.2 & 5.5 & 0.008 \\
\hline Diabetes & 30 & 28 & 33 & 0.02 \\
\hline Hypertension & 70 & 69 & 71 & 0.21 \\
\hline Hyperlipidemia & 64 & 65 & 63 & 0.39 \\
\hline Obesity & 13 & 12 & 15 & 0.03 \\
\hline Coronary artery disease & 92 & 94 & 90 & 0.006 \\
\hline Smoking & 22 & 25 & 19 & 0.002 \\
\hline Alcohol & 2 & 3 & 0.8 & $<0.001$ \\
\hline Heart failure & 16 & 14 & 19 & 0.001 \\
\hline Ventricular arrhythmia & 11 & 12 & 9 & 0.007 \\
\hline Cocaine use & 0.5 & 0.6 & 0.3 & 0.27 \\
\hline Crohn's disease & 0.1 & 0.2 & 0.1 & 0.68 \\
\hline Connective tissue disorder & 0.4 & 0.2 & 0.7 & 0.12 \\
\hline Fibro muscular dysplasia & 0.1 & 0.2 & 0.1 & 0.76 \\
\hline Marfan's syndrome & 0.04 & 0.07 & - & 0.39 \\
\hline Sarcoidosis & 0.2 & 0.2 & 0.3 & 0.37 \\
\hline Rheumatoid arthritis & 1 & 0.8 & 1.2 & 0.54 \\
\hline Giant cell arteritis & 0.05 & - & 0.11 & 0.24 \\
\hline Polycystic kidney disease & 0.05 & 0.09 & - & 0.39 \\
\hline
\end{tabular}

*Continuous variables are expressed as median (IQR). Categorical variables are expressed as \%; **Pearson's $\chi^{2}$ for categorical; linear regression for continuous variables.

\section{Results}

\section{Demographics}

The prevalence of $\mathrm{CD}$ was $0.02 \%(\mathrm{n}=11,255)$ of all hospital admissions reviewed during the year 2009-2010 in United States and was higher in men (57.7\%) compared to women (42.3\%). The mean age was 63.3 years, women were older than men (64.6 years vs. 62.3 years; $\mathrm{p}<0.001$ ), the mean length of stay was 4.5 days and women stayed longer than men (4.9 vs. 4.1 days; $\mathrm{p}=0.001$ ). Chronic conditions prevalent in our study were CAD (92\%), hypertension (70\%), hyperlipidemia $(64 \%)$, diabetes mellitus (30\%) and smoking (22\%). Connective tissue disorders $(0.4 \%)$, sarcoidosis $(0.2 \%)$, Crohn's disease $(0.1 \%)$, rheumatoid arthritis ( $1 \%)$, fibromuscular dysplasia $(0.1 \%)$, giant cell arteritis $(0.05 \%)$, polycystic kidney disease $(0.05 \%)$ and Marfan's syndrome $(0.04 \%)$ were the non-cardiovascular conditions included in our analysis (Table 1).

\section{Primary and secondary outcomes}

In the 11,255 hospital admissions of CD the overall in-patient mortality rate was $4.2 \%$, HF rate was present in $16 \%$ and ventricular arrhythmias were present in $11 \%$ of this population. Women compared to men, had higher mortality (5.5\% vs. $3.2 \%$, respectively, $\mathrm{p}=0.008$ ), more HF ( $19 \%$ vs. $14 \%$, respectively, $\mathrm{p}=0.001$ ) but less ventricular arrhythmia (9\% vs. $12 \%$ in women, $\mathrm{p}=0.007$ ) (Table 1).

We also analyzed the association of various risk factors and comorbidities in predicting mortality in patients with $\mathrm{CD}$. In unadjusted analysis, age (odds ratio [OR] 1.03, 95\% confidence interval [CI] 1.01-1.05, p < 0.001), female sex (OR 1.74, 95\% CI 1.15-2.64, $\mathrm{p}=0.009$ ), HF (OR 2.68, 95\% CI 1.71-4.21, $\mathrm{p}<0.001)$ and ventricular arrhythmias (OR 6.01, 95\% CI 3.85-9.34, p < 0.001) were associated with increased mortality in patients with CD. Interestingly, hyperlipidemia (OR 0.24, 95\% CI 0.15-0.37, p < 0.001) and CAD (OR 0.34, 95\% CI $0.20-0.58, \mathrm{p}<0.001)$ had an inverse relationship in predicting mortality (Table 2). Gender-specific analysis showed hyperlipidemia and ventricular arrhythmia predicted mortality in both men and women. However, age (OR 1.04, 95\% CI 1.01-1.067, $\mathrm{p}=0.003$ ) and HF (OR 3.10, 95\% CI 1.72-5.59, $\mathrm{p}<0.001)$ predicted mortal- 
Table 2. Univariate logistic regression of risk factors predicting mortality.

\begin{tabular}{lcccccc}
\hline Variables & Total & $\mathbf{P}$ & Males & $\mathbf{P}$ & Females & $\mathbf{P}$ \\
\hline Age & 1.03 & $<0.001$ & 1.02 & 0.12 & 1.04 & 0.003 \\
Female & 1.74 & 0.009 & - & - & - & - \\
Length of stay & 1.02 & 0.085 & 1.05 & 0.005 & 0.99 & 0.75 \\
Diabetes mellitus & 0.80 & 0.354 & 0.50 & 0.098 & 1.02 & 0.96 \\
Hypertension & 0.70 & 0.109 & 0.80 & 0.485 & 0.62 & 0.10 \\
Hyperlipidemia & 0.24 & $<0.001$ & 0.17 & $<0.001$ & 0.31 & $<0.001$ \\
Obesity & 0.97 & 0.932 & 0.38 & 0.188 & 1.38 & 0.38 \\
Coronary artery disease & 0.34 & $<0.001$ & 0.24 & $<0.001$ & 0.48 & 0.06 \\
Smoking & 0.53 & 0.038 & 0.55 & 0.160 & 0.55 & 0.18 \\
Heart failure & 2.68 & $<0.001$ & 1.95 & 0.073 & 3.10 & $<0.001$ \\
Ventricular arrhythmia & 6.00 & $<0.001$ & 8.02 & $<0.001$ & 5.29 & $<0.001$ \\
Connective tissue disorder & 2.69 & 0.354 & - & - & 3.21 & 0.29 \\
\hline
\end{tabular}

Table 3. Stepwise multi-model logistic regression of risk factors predicting in-hospital mortality.

\begin{tabular}{|c|c|c|c|c|c|c|}
\hline Model & Total & $\mathbf{P}$ & Males & $\mathbf{P}$ & Females & $\mathbf{P}$ \\
\hline \multicolumn{7}{|l|}{ Age } \\
\hline Demographic risk factors & 1.03 & 0.003 & 1.02 & 0.171 & 1.04 & 0.009 \\
\hline + Traditional risk factors & 1.04 & $<0.001$ & 1.02 & 0.127 & 1.05 & 0.001 \\
\hline+ Non-CV risk factors & 1.04 & $<0.001$ & 1.02 & 0.133 & 1.05 & 0.001 \\
\hline \multicolumn{7}{|l|}{ Ventricular arrhythmia } \\
\hline Demographic risk factors & 7.39 & $<0.001$ & 8.54 & $<0.001$ & 6.27 & $<0.001$ \\
\hline+ Traditional risk factors & 6.03 & $<0.001$ & 8.17 & $<0.001$ & 4.84 & $<0.001$ \\
\hline+ Non-CV risk factors & 6.00 & $<0.001$ & 8.15 & $<0.001$ & 4.73 & $<0.001$ \\
\hline \multicolumn{7}{|l|}{ Heart failure } \\
\hline Demographic risk factors & 2.17 & 0.002 & 1.56 & 0.299 & 2.76 & 0.001 \\
\hline+ Traditional risk factors & 1.87 & 0.015 & 1.33 & 0.538 & 2.45 & 0.006 \\
\hline+ Non-CV risk factors & 1.89 & 0.014 & 1.33 & 0.541 & 2.53 & 0.005 \\
\hline \multicolumn{7}{|l|}{ Hyperlipidemia } \\
\hline Demographic risk factors & 0.20 & $<0.001$ & 0.14 & $<0.001$ & 0.26 & $<0.001$ \\
\hline+ Traditional risk factors & 0.23 & $<0.001$ & 0.16 & $<0.001$ & 0.30 & 0.001 \\
\hline+ Non-CV risk factors & 0.23 & $<0.001$ & 0.16 & $<0.001$ & 0.30 & 0.002 \\
\hline \multicolumn{7}{|l|}{ Coronary artery disease } \\
\hline Demographic risk factors & 0.24 & $<0.001$ & 0.17 & $<0.001$ & 0.32 & 0.006 \\
\hline+ Traditional risk factors & 0.32 & $<0.001$ & 0.29 & 0.007 & 0.40 & 0.030 \\
\hline+ Non-CV risk factors & 0.32 & $<0.001$ & 0.29 & 0.007 & 0.40 & 0.030 \\
\hline
\end{tabular}

CV — cardiovascular; + Step wise addition; Demographic factors: Age, gender and race; Traditional risk factors (or CV risk factors): Diabetes, hypertension, obesity, hyperlipidemia, coronary artery disease, smoking and alcohol use; Non-CV risk factors: C tissue disorder

ity only in females and CAD was associated with decreased mortality only in males (OR $0.24,95 \%$ CI 0.11-0.52, $\mathrm{p}<0.001$ ) (Table 2).

Stepwise multimodel logistic regression showed that age (OR 1.03, 95\% CI 1.01-1.05, $\mathrm{p}=0.001)$, ventricular arrhythmias (OR 5.86, 95\% CI 3.30-10.41, $\mathrm{p}<0.001)$ and HF (OR 1.89, 95\% CI 1.14-3.13, $\mathrm{p}=0.014)$ were independently associated with increased mortality after adjusting for demographic factors, traditional cardiovascular and non-cardiovascular risk factors (Table 3$)$. Hyperlipidemia $(\mathrm{OR}$ $0.23,95 \%$ CI $0.14-0.39, \mathrm{p}<0.001)$ and $\mathrm{CAD}(\mathrm{OR}$ $0.32,95 \%$ CI $0.17-0.58, \mathrm{p}<0.001)$ continued to demonstrate an inverse association in predicting mortality even after adjusting for all risk factors. Gender-specific analysis showed that ventricular 
arrhythmia, hyperlipidemia and CAD predicted mortality in fully adjusted models for both men and women. However, age (OR 1.04, 95\% CI 1.02-1.08, $\mathrm{p}=0.009)$ and HF (OR 2.53, 95\% CI 1.33-4.80, $\mathrm{p}=0.005$ ) predicted mortality only in women and not in men (Table 3 ).

\section{Discussion}

Our analysis is the largest study to date on CD with a prevalence of $\mathrm{CD}(0.02 \%)$ and high mortality rate as compared to previous studies [5, 11-13] which may be attributed to the large sample size and the inclusion of all types of CD. There was an increased prevalence in men compared to women, whereas other studies demonstrated the opposite. This is probably because all types of CD were included our analysis, whereas other studies have included mostly SCD which is more prevalent in younger women with FMD. Our population also had a very low number of patients with FMD, which occurs more commonly in women with SCD [14]. Women had a higher mortality rate $(5.5 \%)$ than males $(3.2 \%)$, something that has been not been described before in a CD population. Not unexpectedly, we identified age, ventricular arrhythmia and $\mathrm{HF}$ as predictors of increased mortality in patients with CD (with advanced female age, increased prevalence of ventricular arrhythmia and HF among females in our study). However, hyperlipidemia and CAD demonstrated an inverse association with predicting mortality. This once again may be explained by the inclusion of all types of CD including atherosclerotic and iatrogenic, which may be associated with lower mortality in general and also be more likely to include patients treated with statins. Treatment with statins may be protective and may be used more frequently in patients with non-SCD since statins are known to reduce peri-procedural myocardial infarction [15], diminish the inflammatory response $[13,14,16]$ and are used for primary $[16,17]$ and secondary prevention $[18,19]$ of cardiovascular death $[20,21]$. Future studies may determine their role in all comers with $\mathrm{CD}$.

\section{Limitations of the study}

Our analysis has several limitations since this is an observational study of a large NIS; it includes all types of $\mathrm{CD}$ where a review of the coronary angiograms was not possible. Previous studies have focused mostly on SCD, which may be a distinct clinical entity more common in women with FMD and postpartum status. Also, the very high percentage of patients with preexisting CAD makes it likely that high numbers of procedure related CD were included in our analysis. Since, the diagnosis of $\mathrm{CD}$ is easier to establish in patients with otherwise unremarkable coronary arteries (i.e. young patients) then in patients with preexisting $\mathrm{CAD}$ where in many cases additional imaging to coronary angiography may be needed to demonstrate a false lumen or hematoma (information that is usually not available from administrative database like NIS), it is essential to have a ICD-9 code for SCD that would help physician scientists to separate the distinct types of dissection. In addition our study excluded patients admitted to the hospital under observational status or those who were discharged home from the emergency room and those patients admitted to any federal hospital (due to absence of information recorded in the NIS database). It is possible that the actual estimate of patients with $\mathrm{CD}$ was greater than that recorded in the database, thereby underestimating the power of the study. Since NIS is an administrative database, diagnostic codes are subject to misclassification and there is no way to determine if secondary diagnoses represented an active problem versus past medical history. The retrospective design of the study can only demonstrate association, and hence causation between the predictors of mortality in CD cannot be determined. Information from relevant studies, such as echocardiograms, coronary angiography, computed tomography angiography, single photon emission computed tomography, and cardiac biomarker data are also not available from the NIS database.

\section{Conflict of interest: None declared}

\section{References}

1. Pretty HC. Dissecting aneurysm of coronary artery in a woman aged 42. BMJ, 1931. 1: 667.

2. Shamloo BK, Chintala RS, Nasur A et al. Spontaneous coronary artery dissection: Aggressive vs. conservative therapy. J Invasive Cardiol, 2010; 22: 222-228.

3. Hendiri T, Bonvini RF, Martin W, Doriot PA, Camenzind E. Acute myocardial infarction due to spontaneous coronary artery dissection. Arch Mal Coeur Vaiss, 2005; 98: 974-978.

4. Hering D, Piper C, Hohmann C, Schultheiss HP, Horstkotte D. Prospective study of the incidence, pathogenesis and therapy of spontaneous, by coronary angiography diagnosed coronary artery dissection. Z Kardiol, 1998; 87: 961-970.

5. Vanzetto G, Berger-Coz E, Barone-Rochette G et al. Prevalence, therapeutic management and medium-term prognosis of spontaneous coronary artery dissection: results from a database of 11,605 patients. Eur J Cardiothorac Surg, 2009; 35: 250-254.

6. Kamineni R, Sadhu A, Alpert JS. Spontaneous coronary artery dissection: report of two cases and a 50-year review of the literature. Cardiol Rev, 2002; 10: 279-284. 
7. Mulvany NJ, Ranson DL, Pilbeam MC. Isolated dissection of the coronary artery: A postmortem study of seven cases. Pathology, 2001; 33: 307-311.

8. Awadalla H, Sabet S, El Sebaie A, Rosales O, Smalling R. Catheter-induced left main dissection incidence, predisposition and therapeutic strategies experience from two sides of the hemisphere. J Invasive Cardiol, 2005; 17: 233-236.

9. Eshtehardi P, Adorjan P, Togni M et al. Iatrogenic left main coronary artery dissection: Incidence, classification, management, and long-term follow-up. Am Heart J, 2010; 159: 1147-1153.

10. Vavuranakis M, Latsios G, Tousoulis D et al. Spontaneous coronary dissection as a cause of acute coronary syndrome: Evidence for non-inflammatory underlying mechanisms. Int J Cardiol, 2007; 114: 24-26.

11. Motreff P, Souteyrand G, Dauphin C, Eschalier R, Cassagnes J, Lusson JR. Management of spontaneous coronary artery dissection: Review of the literature and discussion based on a series of 12 young women with acute coronary syndrome. Cardiology, 2010; 115: 10-18.

12. Vrints CJ. Spontaneous coronary artery dissection. Heart, 2010; 96: 801-808.

13. Butler R, Webster MW, Davies G et al. Spontaneous dissection of native coronary arteries. Heart, 2005; 91: 223-224.

14. Saw J, Poulter R, Fung A, Wood D, Hamburger J, Buller CE. Spontaneous coronary artery dissection in patients with fibromuscular dysplasia: A case series. Circ Cardiovasc Interv, 2012; 5: 134-137.

15. Benjo AM, El-Hayek GE, Messerli F et al. High dose statin loading prior to percutaneous coronary intervention decreases cardiovascular events: A meta-analysis of randomized controlled trials. Catheter Cardiovasc Interv, 2015; 85: 53-60.

16. Downs JR, Clearfield M, Weis S et al. Primary prevention of acute coronary events with lovastatin in men and women with average cholesterol levels: results of AFCAPS/TexCAPS. Air Force/ /Texas Coronary Atherosclerosis Prevention Study. JAMA, 1998; 279: 1615-1622.

17. Shepherd J, Cobbe SM, Ford I et al. Prevention of coronary heart disease with pravastatin in men with hypercholesterolemia. West of Scotland Coronary Prevention Study Group. N Engl J Med, 1995; 333: 1301-1307.

18. Sacks FM, Pfeffer MA, Moye LA et al. The effect of pravastatin on coronary events after myocardial infarction in patients with average cholesterol levels. Cholesterol and Recurrent Events Trial investigators. N Engl J Med, 1996; 335: 1001-1009.

19. The Long-Term Intervention with Pravastatin in Ischaemic Disease (LIPID) Study Group. Prevention of cardiovascular events and death with pravastatin in patients with coronary heart disease and a broad range of initial cholesterol levels. N Engl J Med, 1998; 339: 1349-1357.

20. Amarenco P, Goldstein LB, Szarek M et al.; SPARCL Investigators. Effects of intense low-density lipoprotein cholesterol reduction in patients with stroke or transient ischemic attack: The Stroke Prevention by Aggressive Reduction in Cholesterol Levels (SPARCL) trial. Stroke, 2007; 38: 3198-3204.

21. Byington RP, Davis BR, Plehn JF et al. Reduction of stroke events with pravastatin: the Prospective Pravastatin Pooling (PPP) Project. Circulation, 2001; 103: 387-392.

APPENDIX 1. International Classification of Disease, version 9 (ICD-9) codes used for coronary artery dissection identification.

\begin{tabular}{lc}
\hline Inclusion criteria & ICD-9 codes \\
\hline Coronary artery dissection (any position; primary or all listed diagnosis) & 414.12 \\
\hline
\end{tabular}

APPENDIX 2. International Classification of Disease, version 9 (ICD-9) codes used for co-morbidity identification.

\begin{tabular}{ll}
\hline Co-morbidities* & ICD-9 codes \\
\hline Coronary artery disease & $412,414 . x x$, V458.1, V458.2 \\
Heart failure & $428 . x x$ \\
Hyperlipidemia & $272 . x x$ \\
Obesity & $278 . x x, 793.91$, V85.21-25, V85.30-45, V85.54 \\
Hypertension & $401 . x, 402 . x x, 403 . x x, 404 . x x, 405 . x x, 437.2$ \\
Diabetes mellitus & $249 . x x, 250 . x x, 790 . x x, 791.5,791.6$, V458.5, V539.1, V654.6 \\
Ventricular arrhythmias & $427,427.1,427.4$ \\
Alcohol use & $303 . x x, 305.01,305.02,305.03$ \\
Smoking & $305.1, V 15.82$ \\
Connective tissue disorder & $710 . x x$ \\
Marfan's syndrome & 759.82 \\
Ehlers-Danlos syndrome & 756.83 \\
Fibro muscular dysplasia & 447.8 \\
Sarcoidosis & 135 \\
Crohn's disease & $555 . x$ \\
Cocaine use & $305.60-63,304.20-23$ \\
Premature labor & $644 . x x$ \\
Rheumatoid arthritis & 714.0 \\
Giant cell arteritis & 446.5 \\
Takayasu's disease & 446.7 \\
Polyartertis nodosa & 446.0 \\
Polycystic kidney disease & $753.12,753.13,753.14$ \\
\hline
\end{tabular}

*Primary and all listed diagnosis 